\title{
Chronic cerebrospinal venous insufficiency: current perspectives
}

This article was published in the following Dove Press journal:

Journal of Vascular Diagnostics

3 March 2014

Number of times this article has been viewed

\section{Marian Simka \\ Department of Nursing, College of Applied Sciences, Ruda Śląska, Poland}

Correspondence: Marian Simka 43-245 Studzionka, ul Jedności 20, Poland Tel +48322120498

Fax +483 $2797 \quad 1070$

Email mariansimka@poczta.onet.pl
Abstract: This review summarizes the research to date on chronic cerebrospinal venous insufficiency (CCSVI). CCSVI was initially defined as a clinical syndrome comprising stenoses of the internal jugular and/or azygos veins, characterized by collateral venous outflows and reduced cerebral blood flow, and was found primarily in patients with multiple sclerosis. However, the published evidence on CCSVI is very discordant. Catheter venography studies gave a regular picture, with the majority of patients with multiple sclerosis presenting with demonstrable outflow abnormalities in the veins draining the central nervous system. The prevalence of these lesions was over 50\%, and even higher (about 90\%) when more liberal definition of an abnormality or intravascular sonography was used. Further, the results of magnetic resonance venography studies have been quite consistent, in that stenoses of the internal jugular veins have been found in $25 \%-70 \%$ of patients with multiple sclerosis. In contrast, Doppler sonography studies have revealed CCSVI in $0 \%$ to $100 \%$ of patients. The research is currently suggesting that CCSVI is not a single entity, but rather a group of different anatomic and functional venous abnormalities. Regarding venous outflow from the brain, a patient can present either with diminished inflow to the internal jugular veins resulting from decreased cerebral circulation or with externally compressed or hypoplastic internal jugular veins or stenotic jugular valves. Considering these many faces of CCSVI, it becomes more comprehensible as to why the results of the studies, especially those utilizing Doppler sonography, have been so discordant. Not only were investigators using different diagnostic modalities and distinct protocols, but they were not looking for the same pathology. Since these abnormalities were indeed differently prevalent in patients and healthy controls, the results inevitably became conflicting. This review suggests how future research, preferably using a multimodal approach, should be directed towards clarifying the conflicting results of studies in CCSVI.

Keywords: Doppler ultrasound, jugular vein, magnetic resonance imaging, multiple sclerosis, phlebography, venous malformation

\section{Introduction}

Just a few years ago, Zamboni et al reported that almost all patients with multiple sclerosis can be found to have venous abnormalities in the main veins draining the brain and spinal cord, ie, the internal jugular veins (IJVs) and the azygos vein. They called this syndrome, which was not present in healthy controls, chronic cerebrospinal venous insufficiency (CCSVI). ${ }^{1}$ They also put forward the notion that such a venous pathology may have a primary role in initiating multiple sclerosis, through iron toxicity, chronic ischemia of the brain, or another as yet undiscovered pathway. ${ }^{2}$ This very controversial hypothesis has managed to stoke ongoing debate in the scientific community. However, with a few exceptions, these results have not been replicated 
in subsequent reports by other investigators. Moreover, the results of later studies are very conflicting, with some supporting a strong association between CCSVI and multiple sclerosis, and others finding either an uncertain relationship or no association at all. Even if meta-analyses of these studies suggested a higher prevalence of CCSVI amongst patients with multiple sclerosis in comparison with healthy controls, ${ }^{3-5}$ these discordant reports have added to the confusion regarding this problem rather than explaining it.

This review summarizes the research on CCSVI in an attempt to explain the conflicting results of the studies. Suggestions are also made as to how future research could be directed to clarify the enigma of CCSVI. At the moment, a potential reader can find a number of reviews on this topic. Unfortunately, these papers are not very helpful in understanding the puzzle. While proponents of CCSVI theory claim that the negative studies have been conducted or interpreted improperly, its opponents suggest quite the contrary. Here an attempt is made to explain how these contradictory reports, seen in a bigger picture, are no longer inconsistent. In order to minimize confusion, incompetent jugular valves (ie, allowing backward flow), which seem to be a distinct vascular pathology, are not discussed. Also, since very little is known about the physiology and pathology of the azygos vein system, abnormalities in this venous territory are not discussed in detail either. Instead, the focus is on venous outflow from the brain through the IJVs and vertebral veins.

\section{Current knowledge about the anatomy and physiology of the IJVs}

It is well known that the IJVs constitute a primary outflow route from the brain in the supine position, while in the upright position a substantial part of venous outflow is shifted towards the vertebral veins. ${ }^{6,7}$ Still, rather surprisingly, our knowledge about the anatomy and physiology of these veins is incomplete, especially in the setting of neurologic disease. ${ }^{8}$ Most of the previous studies focused on localization and the diameter of the IJVs, which can be of importance during their catheterization in critically ill or dialyzed patients. It is also known that these veins are valveless, except for the single valve localized just above the junction with the brachiocephalic vein., ${ }^{9,10}$ These jugular valves, when competent, are thought to play an important role in establishing physiological venous outflow from the brain during increased intrathoracic pressure (eg, coughing, Valsalva maneuver, blunt chest injury, and cardiopulmonary resuscitation). It is also known that incompetent jugular valves are prevalent in patients with transient global amnesia, transient monocular blindness, and Alzheimer's disease, ${ }^{11-16}$ and thus may play a role in the pathophysiology of these disorders. There are also reports on other structural abnormalities of the IJVs, including fenestrations, duplications, and ectasia, with uncertain meaning of these anomalies. ${ }^{17,18}$

\section{Results of catheter venography and IVUS}

Catheter venography, respecting its limitations, should be seen as a reference test, since such an angiographic examination is the gold standard for assessment of the morphology of blood vessels and flow abnormalities in other venous territories. ${ }^{19}$ Most of the studies that utilized catheter venography have revealed pathologic venous flow in $95 \%-100 \%$ of patients with multiple sclerosis (Figure 1).,20-27 Importantly, intravascular ultrasound (IVUS) was found to be very helpful as an adjunct to catheter venography. A combination of venography and IVUS provides a comprehensive assessment of venous anatomy, endoluminal structures, and flow. IVUS has revealed even more lesions than catheter venography, since CCSVI seems to be principally an endoluminal disease. ${ }^{21,28-31}$ Given that valvular pathology of the IJV is a predominant

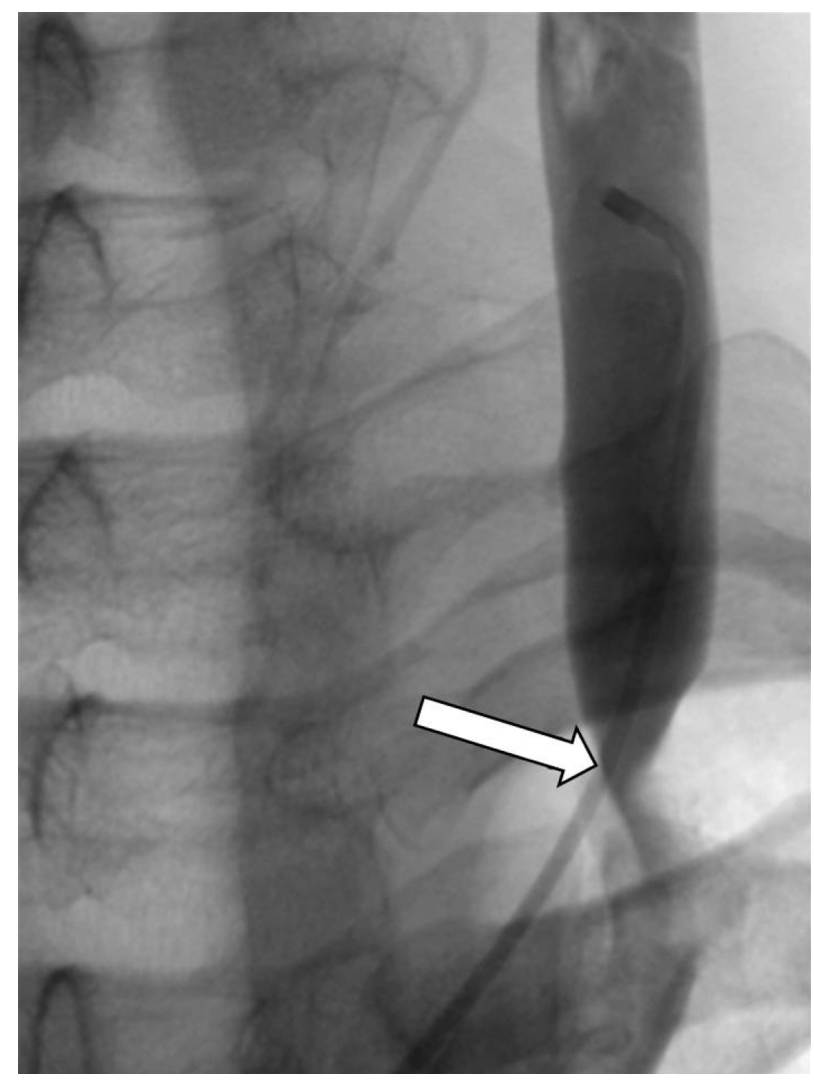

Figure I Stenotic jugular valve (arrow) demonstrated by catheter venography. 


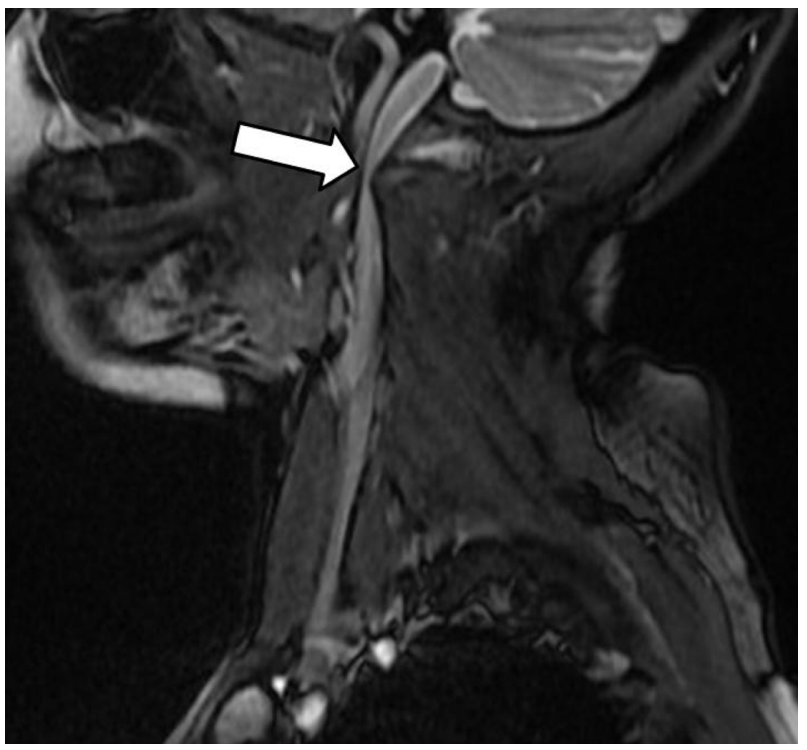

Figure 2 Typical narrowing (arrow) of the internal jugular vein at the level of $\mathrm{Cl}$ demonstrated by magnetic resonance venography (two-dimensional fast imaging employing steady-state acquisition technique)

CCSVI abnormality, an adequate technique for catheter venography is essential. Nondiluted high-viscosity contrast may overshadow tiny endoluminal webs and membranes. ${ }^{19}$

This is probably the reason why investigators who utilized nondiluted contrast found less frequent $(55 \%-70 \%)$ pathology in patients with multiple sclerosis. ${ }^{32,33}$ Also, it should be remembered that catheter venography is inadequate to demonstrate external compression of the IJVs by aberrant muscles, because radiologic contrast injected into the vein,

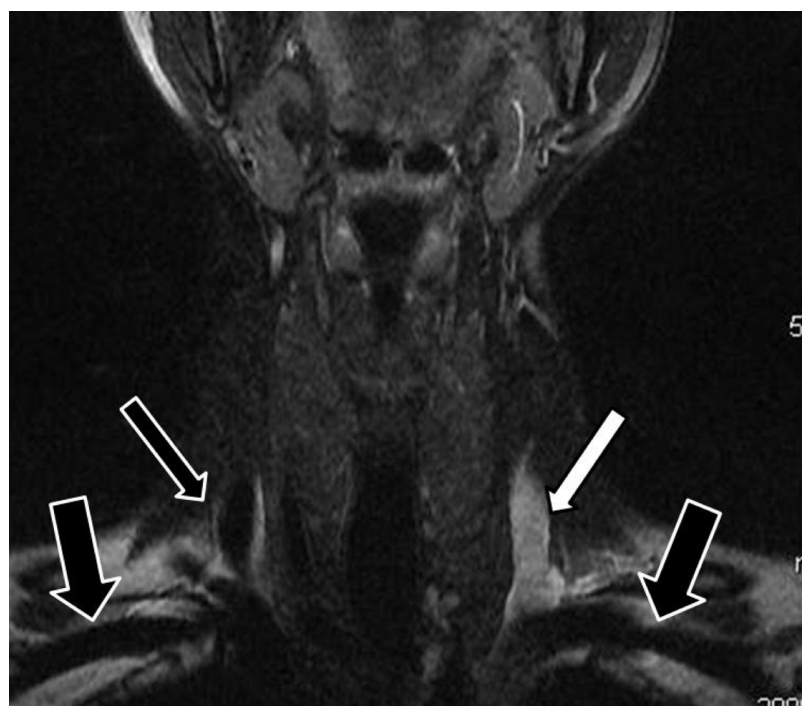

Figure 3 Magnetic resonance venography of patient with multiple sclerosis: fast spin-echo T2-weighted sequences with fat saturation. In this imaging technique, blood vessels with stagnant flow become white. ${ }^{122}$ Normal flow in both subclavian veins (thick arrows) and right internal jugular vein (thin black arrow); stagnant flow in left internal jugular vein (white arrow).

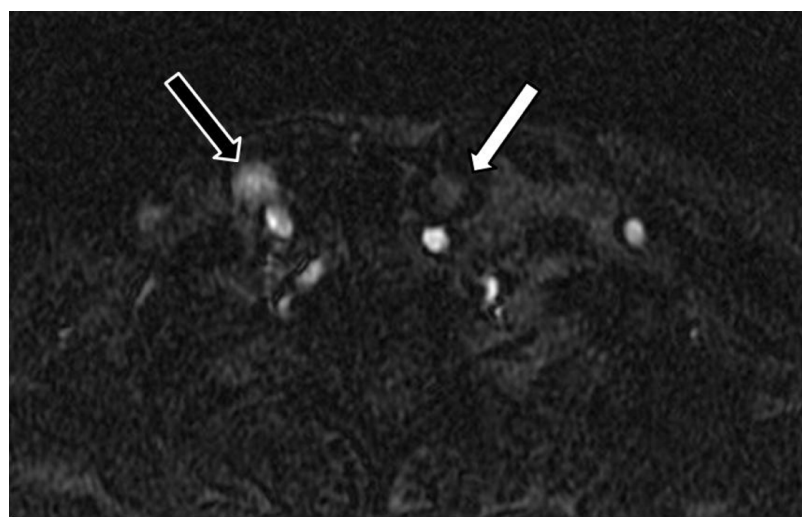

Figure 4 Magnetic resonance venography in a patient with multiple sclerosis: twodimensional time-of-flight imaging. In this technique, blood vessels with normal flow present with bright signal. ${ }^{122}$ Normal flow in right internal jugular vein (black arrow); nearly no signal depicts blood stasis in left internal jugular vein (white arrow).

even under low pressure, can easily reopen a compressed vein. Consequently, such a vein may appear venographically unchanged. ${ }^{34}$ Further, decreased venous flow in the IJVs resulting from poor inflow from cerebral veins cannot be revealed by catheter venography. Similarly, unless an intracranial venogram is performed, atypical anatomy of the cerebral sinuses cannot be demonstrated. Thus, a substantial percentage of CCSVI is likely to be underdiagnosed. Only a few studies have performed catheter venography of the IJVs in healthy controls ${ }^{33}$ and in patients with non-neurological diseases. ${ }^{35}$ Contrary to the "textbook" knowledge, these studies reveal a surprisingly high prevalence of abnormalities (about 70\%), casting doubt on a primary role for CCSVI in the pathogenesis of multiple sclerosis, and also challenging the so-called "negative" CCSVI studies. Unfortunately, precise data regarding the localization and characteristics of venous abnormalities in healthy controls and patients with multiple sclerosis ( 84 versus 65 individuals) were not published in this paper. ${ }^{33}$ Given that it is possible that the lesions were in fact unevenly distributed, it is of particular importance that the authors present this information in their next paper. These data should also be interpreted with caution considering the definition of stenosis applied, ie, 50\% narrowing of the vein relative to the largest normal segment in the supine position.

\section{Results of magnetic resonance venography studies}

Magnetic resonance venography (MRV) is usually considered an objective and not operator-dependent diagnostic modality. Using MRV, the IJV can be evaluated from its origin inside the skull (which cannot be done by ultrasound) to its junction with the brachiocephalic vein. Thus, it might 


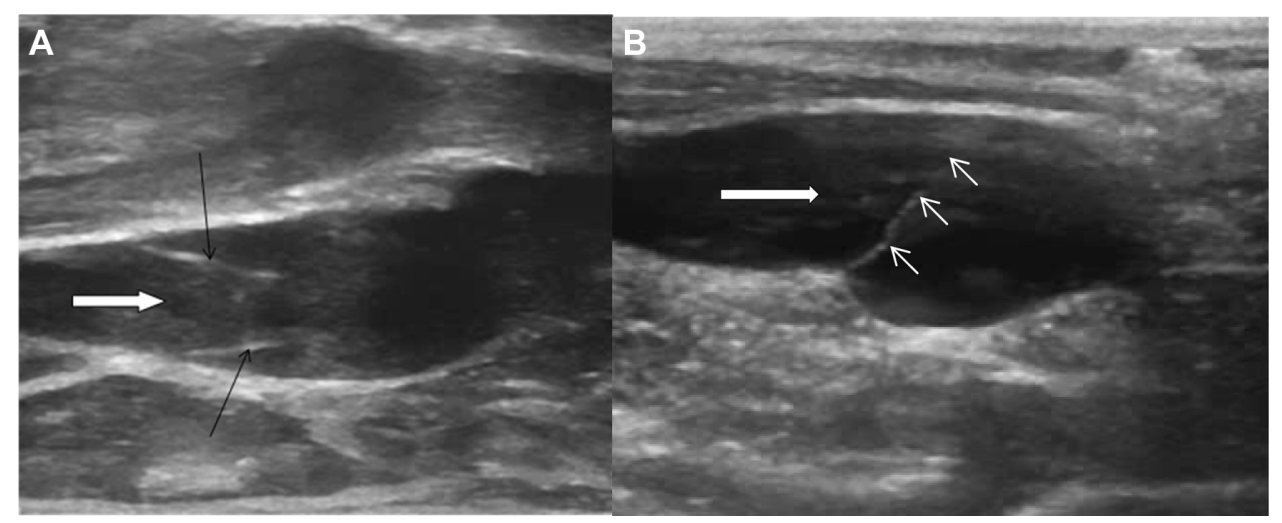

Figure 5 Normal (A) and pathological (B) jugular valve. Thick white arrow indicates flow direction; thin black arrows indicate normal valve leaflets; thin white arrows indicate abnormal valve leaflet forming a membrane obstructing the outflow.

have been expected that research using this technique would give a concise picture. However, the published evidence is quite discordant. While some researchers demonstrated obvious abnormalities, others were unable to demonstrate lesions. MRV is actually not a single imaging technique, but rather a collection of very different diagnostic modalities. Bearing this in mind, it is easier to comprehend differences between the results of the trials. MRV studies on the IJVs can be categorized into three main groups, ie, those looking at morphology of these veins, ${ }^{32,36-40}$ functional magnetic resonance evaluating flow, ${ }^{41}$ and magnetic resonance techniques evaluating both morphology and flow. ${ }^{30,42-44}$ Further, current magnetic resonance imaging is not very good at demonstrating pathology of the jugular valves (catheter venography studies tell us that this is the most common CCSVI abnormality)..$^{25,27-29,45}$ These valves are tiny structures, which are not easily visible on standard magnetic resonance images. Also, artifacts resulting from respiratory and cardiac movements routinely blur the area of the jugular valves, as does gadolinium contrast. Conventional MRV that primarily focuses on the morphology of the vein can easily reveal a narrowing of its upper segment and also a flattening of its middle portion (usually caused by external muscular compression, but also seen in dehydrated patients). However, intraluminal stenoses of the IVJs resulting from structural abnormalities of the valves are not routinely visualized by MRV.

Morphological abnormalities of the IJV, primarily flattening of its upper portion at the level of $\mathrm{C} 1$ (Figure 2) and less frequently stenoses in the lower neck have been found in $25 \%-70 \%$ of patients with multiple sclerosis. ${ }^{30,32,36,37,39,40,44,46}$ Compression of the upper IJV was also found by MRV studies in $15 \%-50 \%$ of healthy individuals. . $^{30,32,37,40,46}$ Further, a similar prevalence and localization of IJV stenoses in healthy controls were revealed by computed tomography angiographic studies. ${ }^{47,48}$ Nonetheless, a pathological role of flattening of the upper IJV remains elusive. Importantly, patients with progressive multiple sclerosis were found to have more magnetic resonance abnormalities of the IJVs than those presenting with a nonprogressive course of disease.$^{37}$ Also, a flattening of the middle part of the IJV was more prevalent in patients with multiple sclerosis than in healthy controls. ${ }^{49}$ The other abnormality that could be revealed by means of MRV was the presence of enlarged venous collaterals in the neck. ${ }^{30,37,39,40,49}$ Such collaterals were found more often in patients with multiple sclerosis than in healthy controls. ${ }^{37,40}$ However, such an increased collateral outflow has also been seen in patients with migraine, ${ }^{41}$ and is probably an anomalous collateral outflow pattern that is not specific for multiple sclerosis. The reported prevalence of magnetic resonance abnormalities depends largely on the definition of pathology used, which may explain differences between the studies. Still, most of the morphological MRV studies did not find substantial differences between patients with multiple sclerosis and healthy controls, irrespective of whether or not they utilized contrastenhanced MRV, noncontrast imaging (such as time-of-flight technique) (Figures 3 and 4), or a combination of these tests. However, it is possible that the researchers were not using optimal imaging protocols. One study that used more sophisticated IJV-dedicated magnetic resonance protocols revealed more stenoses in patients with multiple sclerosis $(55 \%$ and $61 \%)$ than in a control group (5\% and $10 \%) .^{38}$

To date, few magnetic resonance studies evaluating flow in the IJVs and other neck veins have been published. There are several important problems related to proper evaluation of venous flow in the IJVs that have to be solved. For example, pulsatility of venous flow depending on the cardiac cycle and respiratory movements should be considered when interpreting magnetic resonance data. ${ }^{50,51}$ There are some 


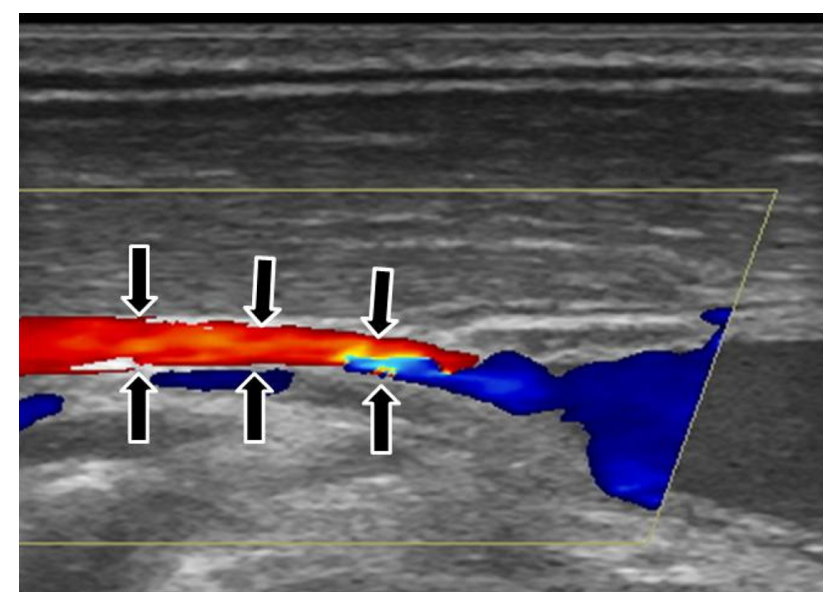

Figure $\mathbf{6}$ Compression of middle part of the internal jugular vein by adjacent muscles.

papers describing IJV flow in healthy people. A phase-contrast magnetic resonance imaging study revealed abnormal flow (strictly unilateral venous cerebral drainage, without any contralateral IJV flow) in $45 \%$ of healthy individuals. ${ }^{51}$ Other researchers studied flow characteristics in the IJVs of patients with multiple sclerosis by means of two-dimensional phase contrast magnetic resonance imaging. They found that stenoses indicated by morphological MRV significantly affected flow, in that those patients with multiple sclerosis diagnosed to have stenoses showed significantly reduced jugular venous flow compared with patients who did not have stenoses. ${ }^{44}$ In addition, localization of stenoses in the lower part of the IJV led to more severe reduction of flow. ${ }^{42,43}$ Interestingly, total arterial flow was not different between multiple sclerosis patients with and without stenoses, ${ }^{42,44}$ so cerebral venous outflow in "stenotic" patients with multiple sclerosis was probably shifted from the IJVs to an alternative venous network. However, it is noteworthy that one study using functional magnetic resonance flow assessment did not reveal a significant difference in flow between patients with multiple sclerosis and healthy controls (the controls did not have statistically significant higher flow volume) ${ }^{52}$ Still, in this study, patients with severe narrowing of the IJV were not assessed separately, as they were in other similar studies. ${ }^{42-44}$

The fact that the IJVs were evaluated only in the supine position (most magnetic resonance units cannot examine standing or sitting patients) appears to be the main limitation of current MRV studies. Doppler sonography, on the contrary, can assess these veins both in the supine and upright body positions. There have been some attempts to evaluate IJVs in sitting patients, ${ }^{46}$ but the methodology is still in its infancy.

\section{Results of Doppler sonographic studies}

Until the seminal paper by Zamboni et al, ${ }^{1}$ most of the sonographic studies of the IJVs evaluated the localization and diameter of these veins. The authors did not observe occluded or severely narrowed veins, and even if they did, such an occlusion was very rare $(1 \%-4 \%$ of occluded IJVs due to thrombosis after many prior cannulations in critically ill or dialyzed patients). ${ }^{53-55}$ Similarly, no occluded or severely narrowed IJVs were found in a study that utilized computed tomography. ${ }^{56}$ Hypoplastic IJVs (cross-sectional area less than $30 \mathrm{~mm}^{2}$ ) were either not seen in healthy people $\mathrm{e}^{57}$ or revealed only in a minority (11.1\%) of subjects. ${ }^{37}$ In another study, small IJVs, defined as having a cross-sectional area less than $40 \mathrm{~mm}^{2}$, were found in $28 \%$ of healthy controls. ${ }^{58}$ There were

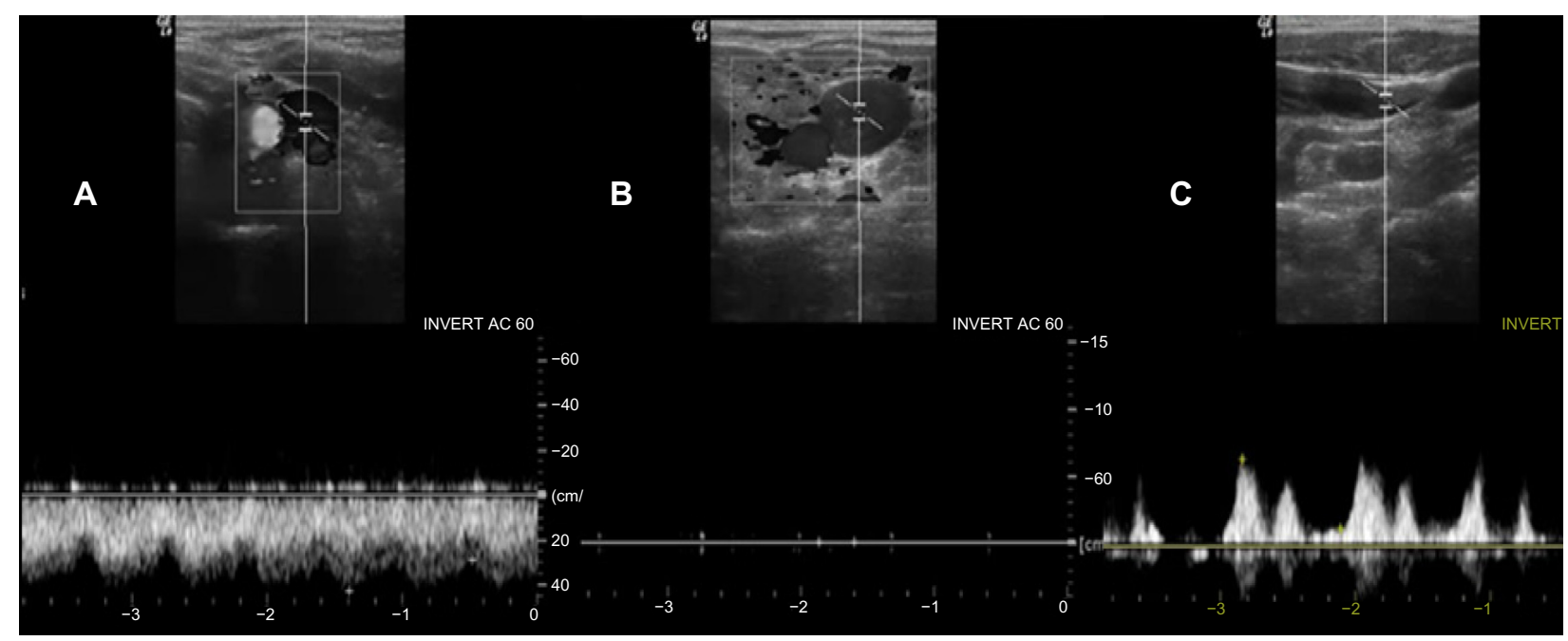

Figure 7 Flow patterns in middle part of the internal jugular vein in the supine position. (A) Normal flow (constant and monodirectional), (B) patent vein but no flow is detected, and (C) anomalous flow (bidirectional, peaks of flow not related to cardiac and respiratory cycles). 
also some studies of jugular valves. Most of the IJVs have a valve localized in the distal portion of the vein, just above the junction with the brachiocephalic vein (Figure 5). Absent jugular valves (in about $10 \%$ of people examined) were seen more often on the left side. ${ }^{9,10}$ In most healthy individuals, the IJVs constitute the main outflow route from the brain in the supine position. Only a minority of healthy people (about $6 \%$ ) present with an alternative cerebral venous outflow pattern. ${ }^{6}$ Alternative outflow pathways through the vertebral veins and deep cervical veins seem to be more prevalent in patients with multiple sclerosis ${ }^{2,36}$ and perhaps also in some other neurologic disorders. ${ }^{41}$ However, an exact prevalence of such anomalies is currently difficult to establish, since no widely accepted criteria of an aberrant outflow pattern exist.

CCSVI is routinely defined using sonographic parameters, the so-called Zamboni's criteria, ie: reflux constantly present in the IJVs and/or vertebral veins with the head in the supine and sitting positions; reflux in the deep cerebral veins; high resolution B-mode evidence of proximal IJV stenosis; flow not detectable in the IJVs and/or vertebral veins despite deep inspirations with the head in the supine and sitting positions; and negative change in the cross-sectional area of the IJV with the head in the supine and sitting positions, denoting the loss of normal postural control. According to Zamboni et al, at least two positive criteria represent pathology, ie, CCSVI, and such a vascular abnormality is strongly associated with multiple sclerosis. ${ }^{1,2,59}$ Other sonographic studies that did not use the Zamboni criteria also revealed pathological venous outflow in patients with multiple sclerosis. One study demonstrated a prolonged cerebral circulation time (defined as the difference in arrival time of the sonographic contrast agent bolus between the carotid artery and the IJV). ${ }^{60}$ Quantitative sonographic assessment revealed abnormal flow volumes in the IJVs of patients with multiple sclerosis. ${ }^{61,62}$ However, flow volume in the IJVs did not differ between healthy controls and patients with mild multiple sclerosis or clinically isolated syndrome. ${ }^{63}$ The prevalence of CCVSI detected in patients with multiple sclerosis either using the original Zamboni criteria or others (differently modified sonographic parameters) varies from $100 \%,{ }^{1,59}$ through about $90 \%,{ }^{64-68}$ $70 \%-80 \%,{ }^{37,69-71} 40 \%-60 \%,{ }^{33,72-80} 10 \%-30 \%,{ }^{58,81-88}$ less than $5 \%{ }^{32,52,89-92}$ to $0 \% .^{35,93-96}$ CCSVI was seen to be more prevalent in older subjects ${ }^{69,71,97}$ and in patients with progressive multiple sclerosis. ${ }^{37,65,78,79,85,88}$ It was rare in patients with clinically isolated syndrome $\mathrm{e}^{72,93,98}$ and less frequent in clinically isolated syndrome than in patients with an established diagnosis of multiple sclerosis. ${ }^{79,80,85}$ Many authors did not find sonographic signs of CCSVI in healthy controls. ${ }^{28,35,52,58,59,72,80,83,87,88,95}$ Some researchers diagnosed this pathology in less than $5 \%$ of healthy individuals tested, ${ }^{90-93}$ while others revealed it more frequently, ie, in $5 \%-10 \%,{ }^{57,62,74,82,85,86,89} 10 \%-40 \%,{ }^{69,70,73,76,79,81,84,97}$ or up to $40 \%-60 \% .^{33,37,75,77}$ However, not a single study has revealed sonographic evidence of CCSVI in the majority of healthy people. A number of studies that compared patients with multiple sclerosis and controls revealed the same prevalence of CCSVI in both groups. ${ }^{35,52,73,75,77,81-84,86,89,90,92,93,95}$ Further, other studies demonstrated a significantly higher prevalence of CCSVI in patients with multiple sclerosis. . $28,37,59,69,70,74,79,85,91$ The prevalence of small IJVs (cross-sectional area less than $40 \mathrm{~mm}^{2}$ ) was similar in patients with multiple sclerosis and healthy controls. ${ }^{58}$ Interestingly, an increased prevalence of sonographic signs of CCSVI was also found in patients with Meniere's disease. ${ }^{99}$

Unfortunately, recent sonographic studies of the IJVs have focused primarily on the potential association of CCSVI with multiple sclerosis, instead of trying to understand how sonographic findings (morphology of the vein and flow characteristics) alter in the setting of impaired jugular drainage. Different patient ages and the clinical characteristics of multiple sclerosis may partially explain the lack of congruent results in the studies. In addition, the majority of authors did not verify the results of Doppler sonography against a more reliable diagnostic test, such as catheter angiography ${ }^{26}$ or IVUS. ${ }^{30,31}$ The researchers assessed the IJVs at different levels, used different definitions of normal and abnormal veins, used different sonographic machines, and the sonographers had very different experience in studying the IJVs (it is obvious that some of them evaluated these veins in a manner similar to that used for examination of the carotid arteries). Some of these studies were blinded, ie, the authors did not know whether they were evaluating a patient with multiple sclerosis or a healthy control, ${ }^{33,37,69,73,76,79-81,84,86,89,90,93,100}$ while other trials were not designed in this way. However, blinding did not appear to be very helpful. The prevalence of CCSVI in patients with multiple sclerosis in blinded studies, although not as high as in nonblinded ones, was also very different, ie, $70 \%-80 \%,{ }^{37,69} 40 \%-60 \%,{ }^{33,73,76,79,80}$ only $10 \%-30 \%,{ }^{81,84,86}$ less than $5 \%{ }^{89,90}$ and even $0 \%{ }^{93}$

\section{Why were the results of Doppler sonography studies so inconsistent?}

While the high incompatibility of studies on CCSVI may, to some degree, explain why the results are so contrasting, the different protocols used still do not provide a full answer. It is well known that Doppler sonography is a very operator-dependent 
test. This imaging technique is far more accurate when examining arteries, where high-velocity flow facilitates the assessment. Testing veins with low-velocity flows driven by low-pressure gradients is far more difficult, perhaps with the exception of detection of thrombus. It was also known that an individual in a mildly dehydrated state (such as after fasting overnight) is more likely to meet sonographic criteria for CCSVI, and that in the majority of such individuals an abnormal sonographic pattern is no longer seen after appropriate rehydration. ${ }^{101}$ Similarly, in one study of a number of individuals, the initial diagnosis of CCSVI could not be reconfirmed at follow-up. Also, initially CCSVI-negative patients later presented with sonographic signs of CCSVI. ${ }^{87}$ Further, head rotation can significantly affect flow in the IJVs, especially in patients with multiple sclerosis, ${ }^{57}$ which can be a source of inadequate Doppler sonography results. Appropriate assessment of the IJVs and other neck veins, with no generally accepted sonographic protocol for such an examination, is a real challenge. There are also other problems that diminish the accuracy of Doppler sonography in this area. CCSVI seems to be primarily a hemodynamic disorder. In other venous territories, hemodynamic abnormalities can be assessed sonographically using tests that increase or decrease flow or evoke reflux, eg, manual compression, the Valsalva maneuver, and the Paraná maneuver. Still, no such testing is possible to change cerebral venous outflow without potentially doing harm to the patient. Reflux in the IJV during the Valsalva maneuver is a very distinct abnormality, and not related to CCSVI (as we currently define this clinical entity). It is also known that evoking jugular reflux may result in such neurological sequelae as transient global amnesia or transient monocular blindness. ${ }^{11-15}$

Despite all the above mentioned limitations of Doppler sonography, the results of the studies in CCSVI should theoretically be more congruent. Some researchers have reported good reproducibility of sonography in detecting CCSVI. ${ }^{102,103}$ Of note, reproducibility has been shown to be better between trained sonographers. ${ }^{103}$ However, others found that, for some CCSVI criteria (reflux in deep cerebral veins and no flow detectable in the IJVs or vertebral veins), the agreement between sonographers was no greater than chance. Agreement for other sonographic criteria was also very low. ${ }^{100}$

In contrast with the discordant results of Doppler sonographic studies, catheter venography trials gave a more regular picture, with the majority of patients with multiple sclerosis showing demonstrable outflow abnormalities in the IJVs. The prevalence of these lesions was over $50 \% 0^{32,33}$ and was even higher (about 90\%) when more liberal definition of an abnormality or IVUS were used. ${ }^{1,20-27,104}$ Similarly, the results of MRV studies were quite consistent, ie, IJV stenoses were found in $25 \%-70 \%$ of patients with multiple sclerosis, ${ }^{30,32,36,37,39,40,44,46,49}$ which is comparable with the results of catheter venography. A lower frequency of IJV abnormalities revealed by MRV probably resulted from the fact that this imaging modality cannot precisely assess the jugular valve, which is the most common site of CCSVI pathology. Also, the presence of enlarged venous collaterals in the neck in a patient with multiple sclerosis revealed by means of $\mathrm{MRV}^{30,37,39,40,49}$ suggests impaired outflow through the principal route, ie, the IJVs. Conversely, a number of sonographic studies were either unable to demonstrate a single vascular lesion ${ }^{35,93-96}$ or they found abnormalities in a minority of patients with multiple sclerosis. ${ }^{32,52,58,81-92}$ Thus, if currently used sonographic criteria for CCSVI were reliable, most of the studies should have revealed abnormalities as frequently as catheter venography did, ie, over $50 \%$ of multiple sclerosis patients with significant lesions and about $90 \%$ with minor anomalies. However, only some studies revealed such a prevalence in patients with multiple sclerosis. Obviously, current sonographic CCSVI criteria, ${ }^{1,2,58}$ even those that have been recently modified, ${ }^{105}$ are not good enough to evaluate patients correctly. ${ }^{26,76}$ However, some sonographers, even in blinded studies, have been able to reveal a frequency of CCSVI that was probably roughly correct, ${ }^{37,79,80}$ while others have reported an unreliably low prevalence of CCSVI. ${ }^{86,89,90}$

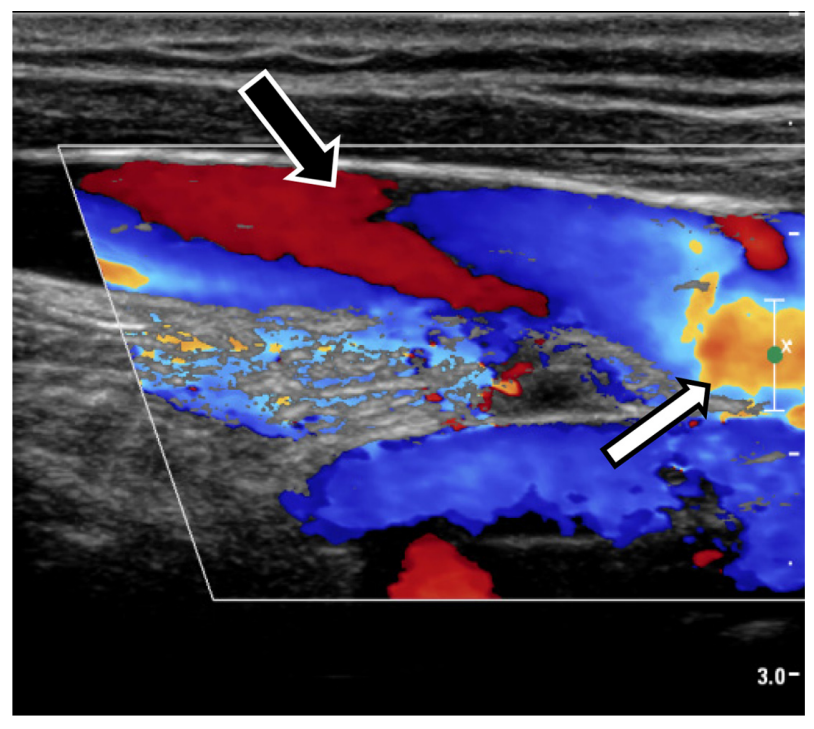

Figure 8 Opposite flow directions in the internal jugular vein demonstrated by color Doppler sonography (black arrow). Red and blue colors are not resulting from the so-called aliasing (white arrow is pointing to a region of aliasing on the right side of figure). Such a phenomenon of different colors revealed by sonography was either interpreted as reflux, or monodirectional vortical flow, or an artifact resulting from improper angle of insonation. However, it is possible that different colors are actually the manifestation of separated flow regions and development of Lagrangian coherent structures. 
Looking more closely at the methodologies of these studies, one should conclude that even if the authors performed the studies properly, their interpretation of the sonographic findings was different. The only objective parameter in the current set of CCSVI criteria ${ }^{59,105}$ is the difference in IJV cross-sectional area between the supine and upright positions ( $\triangle \mathrm{CSA}$ ), which is assumed to be pathological if it is zero or negative. However, this parameter is actually not different between healthy and abnormal IJVs. ${ }^{26}$ It has recently been suggested that the flow volume in the IJV should be assessed perhaps instead of the $\triangle \mathrm{CSA}$, and assumed to be pathological if it is higher in the upright position than in the supine position. ${ }^{106}$ There is also much controversy surrounding sonographic study of the cerebral veins. Given that magnetic resonance perfusion studies revealed abnormal flow in the cerebral veins of patients with multiple sclerosis, ${ }^{107}$ sonographic examination should also find an anomalous flow pattern, at least in some of these patients. However, detection of reflux in the deep cerebral veins is very difficult and requires considerable expertise, so it is likely that only some of the published studies are trustworthy and that others should be discussed with caution. Other sonographic criteria, such as stenosis, reflux in the IJV, and no flow detectable in the $\mathrm{IJV}^{59}$ are far less objective. Importantly, the description of these criteria in current guidelines is not precise, ${ }^{105}$ allowing liberal interpretation of the test. Consequently, the same patient can be diagnosed CCSVI-positive or CCSVInegative ${ }^{90,100}$ using the same sonographic criterion but a different interpretation. Which approach to the unraveling of Doppler sonography is actually correct? Let us summarize what have we learned about venous outflow from the brain during the last few years.

CCSVI was initially defined as a clinical syndrome comprising stenoses of the jugular and/or azygos veins, characterized by collateral venous outflow and reduced cerebral blood flow. ${ }^{1,2,108,109}$ However, we now know that CCSVI, defined as an anomalous outflow from the brain and spinal cord, is not a single entity, but rather a group of different anatomic and functional abnormalities, that in some individuals even come together. In a case of pathological venous outflow from the brain, some patients present with diminished inflow to the IJV resulting from decreased cerebral venous flow, primarily caused by neurologic disease (eg, reduced cerebral metabolism, brain atrophy), from atypical anatomy of the cerebral sinuses (such as hypoplasia of one or both of transversal and/or sigmoid sinuses), ${ }^{110,111}$ or a combination of these. In some individuals, one or both IJVs are found to be externally compressed, usually by aberrant adjacent muscles (Figure 6), ${ }^{34,112}$ arteries, ${ }^{113}$ or bony structures..$^{30,32,37,40,46-48}$ Such compression may also result

Table I Prevalence of particular abnormalities resulting in pathological flows in the IJVs in patients with multiple sclerosis, patients with other neurologic diseases, and healthy controls, according to published data*

\begin{tabular}{|c|c|c|c|}
\hline IJV abnormality & Patients with MS & $\begin{array}{l}\text { Patients with other } \\
\text { neurologic disease }\end{array}$ & Healthy individuals \\
\hline Reduced cerebral venous outflow & $\begin{array}{l}\text { Prevalence unknown, probably } \\
\text { more frequent in MS patients with } \\
\text { longstanding disease }\end{array}$ & Prevalence unknown & $\begin{array}{l}\text { Prevalence unknown, most } \\
\text { likely very rare }\end{array}$ \\
\hline $\begin{array}{l}\text { Atypical anatomy of transverse } \\
\text { and sigmoid sinuses }\end{array}$ & $\begin{array}{l}\text { Prevalence unknown, but some } \\
\text { papers suggest that it may be as } \\
\text { high as } 20 \%^{119}\end{array}$ & $\begin{array}{l}\text { Prevalence unknown, probably quite } \\
\text { common in headache patients }{ }^{120} \text { and } \\
\text { those with Parkinson's disease }\end{array}$ & $\begin{array}{l}\text { Prevalence unknown, may be } \\
\text { as high as } 5 \%-30 \%^{51,111,119}\end{array}$ \\
\hline $\begin{array}{l}\text { External compression of upper } \\
\text { part of IJV }(\mathrm{Cl}, \mathrm{C} 2 \text { vertebrae } \\
\text { and/or digastric muscle) }\end{array}$ & Prevalence unknown & Prevalence unknown & $\begin{array}{l}\text { I5\%-50\%, depending on } \\
\text { definition of stenosis }{ }^{30,32,37,40,46-48}\end{array}$ \\
\hline $\begin{array}{l}\text { External compression of middle } \\
\text { part of IJV (weak venous wall } \\
\text { or omohyoid muscle) }\end{array}$ & $\begin{array}{l}\text { Prevalence } \sim 30 \% \text {, often in combination } \\
\text { with jugular valve pathology } y^{57}\end{array}$ & Prevalence unknown & $\begin{array}{l}\text { Prevalence unknown, probably } \\
\text { uncommon }\end{array}$ \\
\hline $\begin{array}{l}\text { Hypoplastic IJV (cross-sectional } \\
\text { area }<30 \mathrm{~mm}^{2} \text { ) }\end{array}$ & Probably $\sim 10 \%^{57}$ & Prevalence unknown & Rare $^{57}$ \\
\hline Stenotic jugular valve & $\begin{array}{l}\text { Hemodynamically relevant stenosis } \\
\text { of the valve seen in } \sim 70 \% \text { of patients, } \\
\text { mild stenoses in } \sim 95 \% \text { (results of } \\
\text { catheter venography and IVUS) }{ }^{1,20-27,32,33}\end{array}$ & Prevalence unknown & $\begin{array}{l}\text { Prevalence unknown, most } \\
\text { likely common (about 70\%) }\end{array}$ \\
\hline Thrombotic occlusion of IJV & $\begin{array}{l}\text { Rare (may be seen in patients who } \\
\text { have undergone endovascular } \\
\text { treatment for CCSVI) }\end{array}$ & $\begin{array}{l}\text { Prevalence unknown, most } \\
\text { likely very rare }\end{array}$ & Extremely rare \\
\hline
\end{tabular}

Note: *These numbers should be interpreted with caution, since most of the studies cited have not been primarily designed to evaluate such prevalences. Abbreviations: CCSVI, chronic cerebrospinal venous insufficiency; IVUS, intravascular ultrasound; IJV, internal jugular vein; MS, multiple sclerosis. 
from reduced rigidity of the venous wall that, for reasons not presently well understood, perhaps altered composition of collagen fibers, is seen in some patients with multiple sclerosis. ${ }^{57,114}$ Further, some patients are found to have hypoplastic IJVs. ${ }^{57,114}$ Still, the pathology that is most often seen is a stenotic “over-competent" jugular valve. ${ }^{25,27-29,45,57,66,77}$ Such a pathological valve has been found in $70 \%-90 \%$ of patients with multiple sclerosis and also in many healthy controls. ${ }^{33,35}$ Considering these many faces of CCSVI, it becomes more comprehensible why the results of prevalence studies have been so discordant. Not only were the authors using different diagnostic modalities and distinct protocols, but (probably more importantly) they were not actually looking for the same pathology. Since these abnormalities were indeed differently prevalent between patients and healthy controls (Table 1), the results were inevitably conflicting.

The next issue is how to improve the accuracy of Doppler sonography and to construct a more appropriate set of sonographic criteria that would allow better discrimination between a pathological IJV and a healthy vein. It is well known that a particular vascular abnormality can affect sonographic findings in other veins. By extrapolation, sonographic examination is likely to reveal a similar phenomenon in a case of pathological flow in the IJV. However, it is also possible that in this unique venous territory (most of the veins are valveless, with negative hydrostatic pressure in the upright position), a peculiar sonographic pattern will be revealed (Figure 7). Table 2 summarizes how structural and functional abnormalities could theoretically affect the results of sonographic investigation. Given that an individual can present with more than one abnormality, a combination may result in an even more atypical outflow pattern. Sonographic abnormalities accompanying stenotic jugular valves seem to be of particular interest. These can probably be categorized into two main groups (very likely with no strict boundary between them), ie, stenotic valves that do not significantly restrict flow and valves that severely reduce outflow. A functional MRV study revealed significant IJV flow impairment in $46 \%$ of 200 patients with

Table 2 Doppler sonography findings that should accompany abnormalities in the JJ*

\begin{tabular}{lll}
\hline IJV abnormality & Doppler sonography findings & Remarks \\
\hline Reduced cerebral venous outflow & Decreased flow velocity in both IJVs & Probably in many cases reduced flow will not \\
& Collapse of one or both IJVs due to decreased & be detected by standard Doppler sonography, \\
& transmural pressure & since the vein can adjust to diminished \\
flow through shrinking; quantitative flow & assessment should be helpful &
\end{tabular}

Atypical anatomy of transverse and sigmoid sinuses

External compression of upper part of the IJV (CI, C2 vertebrae and/or digastric muscle)

External compression of middle part of the IJV (weak venous wall or omohyoid muscle)

Hypoplastic IJV

Stenotic jugular valve (minor stenosis)

Stenotic jugular valve (major stenosis)

Thrombotic occlusion of IJV
Extremely asymmetric flow in the IJVs or no flow in one IJV

If unilateral: extremely asymmetric flow in the JVs or no flow in one IJV

If bilateral: predominant outflow through vertebral veins in the supine body position Narrowing of the middle part of IJV (rather ellipsoid than pinpoint), which may disappear during such maneuvers as opening of the mouth or head rotation Collateral flow through external jugular vein, thyroid veins, and other cervical veins

Small diameter of the IJV that does not increase during the Valsalva maneuver The vein can be completely collapsed with the presence of collateral outflow network Increased flow velocity at the level of jugular valve, with steady flow in the middle part of the IJV

Either a patent vein with no flow detectable or patent vein with regions characterized by different flow velocities and flow direction or the vein that is completely collapsed Typical signs of venous thrombosis: no flow, noncompressible vein, filling defect inside the vein, with echogenicity depending on age of thrombus
In some cases flow can normalize with head rotation

Narrowing may diminish with Valsalva maneuver

In some cases collateral flow through external jugular vein, thyroid veins and other cervical veins can be seen In some cases collateral flow through external jugular vein, thyroid veins, and other cervical veins can be seen

In some cases collateral flow through external jugular vein, thyroid veins, and other cervical veins can be seen

Note: *Importantly, these are theoretical conjectures that should be validated by future research.

Abbreviation: IJV, internal jugular vein. 
multiple sclerosis. ${ }^{44}$ This abnormal flow was not seen in healthy controls studied by the same authors. ${ }^{43}$ Importantly, flow restriction demonstrated by MRV studies ${ }^{42-44}$ was less frequent than the prevalence of jugular abnormalities found in catheter venography studies (70\%-90\%)..$^{1,20-27,32,33}$ This favors the idea that jugular valves, even if stenotic, can affect flow differently. In the case of a jugular valve with minor stenosis, flow in its proximity is likely to follow the classic laws of fluid mechanics. ${ }^{115}$ Consequently, an increased flow velocity at the level of the valve and decreased flow velocity in the middle segment of the IJV (where the vein is usually wider) should be observed. In a case of hemodynamically relevant stenosis, especially if the valve is asymmetrically shaped, it is likely that the flow would be extremely turbulent. Today, there is a general consensus that in a case of turbulent flow, in addition to the chaotic motion of the fluid (in this case blood, which is a non-Newtonian fluid), there is a development of physical barriers, ie, so-called Lagrangian coherent structures. These structures allow more orderly flow behavior and divide dynamically distinct regions of fluid flow. Thus, the blood on either side of such a Lagrangian coherent structure can be both kinematically and dynamically distinct. Under such conditions, flow recirculation, stagnation, and separation can occur. ${ }^{116}$ It is likely that "reflux", that has been demonstrated in many CCSVI studies, is actually a manifestation of separated flow regions in the IJVs. An IVUS study has already described intraluminal "hyperechoic filling defects" in the area of the jugular valve in patients with multiple sclerosis. ${ }^{30}$ Most likely, these structures were not thrombi (no thrombi were detected by any of the venography studies), but rather separate regions of static blood. Such separate regions, characterized by lowvelocity flows, have already been demonstrated in abdominal and cerebral aneurysms. ${ }^{116}$ It is likely that they may also be found in other blood vessels with turbulent flow. ${ }^{116}$ The nonNewtonian nature of blood probably favors such a flow separation. Importantly, Lagrangian coherent structures are not of an anatomic nature. Although real, they are mathematical constructs and in many cases will not be revealed by catheter venography. Radiologic contrast has physical properties that are different from those of the blood, and outflow of injected contrast is usually less turbulent. Development of Lagrangian coherent structures after contrast injection in most cases would require a different geometry of the vein and its valve. However, Doppler sonography should easily reveal regions with different flow velocity and opposite flow directions (Figure 8). Demonstration of such different regions is likely to be a sign of flow separation and the restriction caused by the jugular valve or other intraluminal abnormalities (eg, flaps or webs) of the IJV. Future research, preferably using a multimodal approach, ${ }^{30,117,118}$ should validate these theoretical conjectures, which are summarized in Table 2. Hopefully, using such studies, a useful protocol for sonographic assessment of the IJVs could be designed.

\section{Disclosure}

The author reports no conflict of interest in this work.

\section{References}

1. Zamboni P, Galeotti R, Menegatti E, et al. Chronic cerebrospinal venous insufficiency in patients with multiple sclerosis. $J$ Neurol Neurosurg Psychiatry. 2009;80(4):392-399.

2. Zamboni P, Galeotti R. The chronic cerebrospinal venous insufficiency syndrome. Phlebology. 2010;25(6):269-279.

3. Lane T. Systematic review of sonographic chronic cerebrospinal venous insufficiency findings in multiple sclerosis. Phlebolymphology. 2012;20(1):26.

4. Laupacis A, Lillie E, Dueck A, et al. Association between chronic cerebrospinal venous insufficiency and multiple sclerosis: a metaanalysis. Can Med Assoc J. 2011;183:E1203-E1212.

5. Thapar A, Lane T, Nicholas R, et al. Systematic review of sonographic chronic cerebrospinal venous insufficiency findings in multiple sclerosis. Phlebology. 2011;26(8):319-325.

6. Doepp F, Schreiber SJ, von Münster T, Rademacher J, Klingebiel R, Valdueza JM. How does the blood leave the brain? A systematic ultrasound analysis of cerebral venous drainage patterns. Neuroradiology. 2004;46(7):565-570.

7. Gisolf J, van Lieshout JJ, van Heusden K, Pott F, Stok WJ, Karemaker JM. Human cerebral venous outflow pathway depends on posture and central venous pressure. $J$ Physiol. 2004;560 Pt 1:317-327.

8. Zivadinov R, Chung CP. Potential involvement of the extracranial venous system in central nervous system disorders and aging. $B M C$ Med. 2013;11:260.

9. Lepori D, Capasso P, Fournier D, Genton CY, Schnyder P. Highresolution ultrasound evaluation of internal jugular valves. Eur Radiol. 1999;9(6):1222-1226.

10. Valecchi D, Bacchi D, Gulisano M, et al. Internal jugular valves: an assessment of prevalence, morphology and competence by color Doppler echography in 240 healthy subjects. Ital J Anat Embryol. 2010;115(3): 185-189.

11. Agosti C, Borroni B, Akkawi NM, Padovani A. Cerebrovascular risk factors and triggers in transient global amnesia patients with and without jugular valve incompetence: results from a sample of 243 patients. Eur Neurol. 2010;63(5):291-294.

12. Chung CP, Hsu HY, Chao AC, Cheng CY, Lin SJ, Hu HH. Jugular venous reflux affects ocular venous system in transient monocular blindness. Cerebrovasc Dis. 2010;29(2):122-129.

13. Chung $\mathrm{CP}, \mathrm{Hu} \mathrm{HH}$. Jugular venous reflux. J Med Ultrasound. 2008;16(3):210-222.

14. Fisher J, Vaghaiwalla F, Tsitlik J, et al. Determinants and clinical significance of jugular venous valve competence. Circulation. 1982;65(1): 188-196.

15. Hsu HY, Chao AC, Chen YY, et al. Reflux of jugular and retrobulbar venous flow in transient monocular blindness. Ann Neurol. 2008;63(2): 247-253.

16. Chung CP, Beggs C, Wang PN, et al. Jugular venous reflux and white matter abnormalities in Alzheimer's disease: a pilot study. J Alzheimer Dis. November 8, 2013. [Epub ahead of print.]

17. Rossi A, Tortori-Donati P. Internal jugular vein phlebectasia and duplication: case report with magnetic resonance angiography features. Pediatr Radiol. 2001;31(2):134. 
18. Towbin AJ, Kanal E. A review of two cases of fenestrated internal jugular veins as seen bycomputed tomography angiography. AJNR Am J Neuroradiol. 2004;25(8):1433-1434.

19. Simka M, Hubbard D, Siddiqui AH, et al. Catheter venography for the assessment of internal jugular veins and azygos vein: position statement by expert panel of the International Society for Neurovascular Disease. Vasa. 2013;42(3):168-176.

20. Denislic M, Milosevic Z, Zorc M, Ravnik IZ, Mendiz O. Disability caused by multiple sclerosis is associated with the number of extracranial venous stenoses: possible improvement by venous angioplasty. Results of a prospective study. Phlebology. 2013;28(7):353-360.

21. Ferral H, Behrens G, Tumer Y, Riemenschneider M. Endovascular diagnosis and management of chronic cerebrospinal venous insufficiency: retrospective analysis of 30-daz morbidity and mortality in 95 consecutive patients. AJR Am J Roentgenol. 2013;200(6): 1358-1364.

22. Ludyga T, Kazibudzki M, Simka M, et al. Endovascular treatment for chronic cerebrospinal venous insufficiency: is the procedure safe? Phlebology. 2010;25(6):286-295.

23. Petrov I, Grozdinski L, Kaninski G, Iliev N, Iloska M, Radev A. Safety profile of endovascular treatment for chronic cerebrospinal venous insufficiency in patients with multiple sclerosis. $J$ Endovasc Ther. 2011;18(3):314-323

24. Petrov I, Grozdinski L, Martinov I, Iliev N, Kaninski G. [Endovascular diagnostics and therapy of chronic cerebrospinal venous insufficiency]. Gazz Med Ital Arch Sci Med. 2012;171(6):755-765. Italian.

25. Simka M, Latacz P, Ludyga T, et al. Prevalence of extracranial venous abnormalities: results from a sample of 586 multiple sclerosis patients. Funct Neurol. 2011;26(4):197-203.

26. Simka M, Ludyga T, Latacz P, Kazibudzki M. Diagnostic accuracy of current sonographic criteria for the detection of outflow abnormalities in the internal jugular veins. Phlebology. 2013;28(6):285-292.

27. Veroux P, Giaquinta A, Perricone D, et al. Internal jugular veins outflow in patients with multiple sclerosis: a catheter venography study. J Vasc Intervent Radiol. 2013;24(12):1790-1797.

28. Al-Omari MH, Al-Bashir A. Internal jugular vein valve morphology in the patients with chronic cerebrospinal venous insufficiency (CCSVI); angiographic findings and schematic demonstrations. Rev Recent Clin Trials. 2012;7(2):83-87.

29. Karmon Y, Zivadinov R, Weinstock-Guttman B, et al. Comparison of intravascular ultrasound with conventional venography for detection of extracranial venous abnormalities indicative of chronic cerebrospinal venous insufficiency. J Vasc Interv Radiol. 2013;24(10) 1487-1498. e1.

30. Zivadinov R, Karmon Y, Dolic K, et al. Multimodal noninvasive and invasive imaging of extracranial venous abnormalities indicative of CCSVI: results of the PREMiSe pilot study. BMC Neurol. 2013;13(1):151.

31. Scalise F, Farina M, Manfredi M, Auguadro C, Novelli E. Assessment of jugular endovascular malformations in chronic cerebrospinal venous insufficiency: colour-Doppler scanning and catheter venography compared with intravascular ultrasound. Phlebology. 2013;28(8): 409-417.

32. Brod SA, Kramer LA, Cohen AM, et al. Chronic cerebrospinal venous insufficiency: masked multimodal imaging assessment. Mult Scler. 2013;19(11):1499-1507.

33. Traboulsee AL, Knox KB, Machan L, et al. Prevalence of extracranial venous narrowing on catheter venography in people with multiple sclerosis, their siblings and unrelated healthy controls: a blinded, casecontrol study. Lancet. 2014;383(9912):138-145.

34. Simka M, Majewski E, Fortuna M, Zaniewski M. Internal jugular vein entrapment in a multiple sclerosis patient. Case Rep Surg. 2012;2012:293568.

35. McAuliffe W, Kermode AG. Mystery of chronic cerebrospinal venous insufficiency: identical venographic and ultrasonographic findings in patients with MS and controls. AJNR Am J Neuroradiol. 2013;34(7): 1370-1374.
36. Doepp F, Würfel JT, Pfueller CF, et al. Venous drainage in multiple sclerosis: a combined MRI and ultrasound study. Neurology. 2011;77(19): 1745-1751.

37. Dolic K, Marr K, Valnarov V, et al. Intra- and extra luminal structural and functional venous anomalies in multiple sclerosis as evidenced by 2 noninvasive imaging techniques. AJNR Am J Neuroradiol. 2012;33(1):16-23.

38. Rahman MT, Sethi SK, Utriainen DT, Hewett JJ, Haacke EM. A comparative study of magnetic resonance venography techniques for the evaluation of the internal jugular veins in multiple sclerosis patients. Magn Reson Imaging. 2013;31(10):1668-1676.

39. Zaharchuk G, Fischbein NJ, Rosenberg J, Herfkens RJ, Dake MD. Comparison of MR and contrast venography of the cervical venous system in multiple sclerosis. Am J Neuroradiol. 2011;32(8):1482-1489.

40. Zivadinov R, Lopez-Soriano A, Weinstock-Guttman B, et al. Use of MR venography for characterization of the extracranial venous system in patients with multiple sclerosis and healthy control subjects. Radiology. 2011;258(2):562-570.

41. Ertl-Wagner B, Koerte I, Kümpfel T, et al. Non-specific alterations of craniocervical drainage in multiple sclerosis revealed by cardiac-gated phase-contrast MRI. Mult Scler. 2012;18(7):1000-1007.

42. Feng W, Utriainen D, Trifan G, et al. Characteristics of flow through the internal jugular veins and cervical $\mathrm{C} 2 / \mathrm{C} 3$ and $\mathrm{C} 5 / \mathrm{C} 6$ levels for multiple sclerosis patients using MR phase contrast imaging. Neurol Res. 2012;34(8):802-809.

43. Feng W, Utriainen D, Trifan G, Sethi S, Hubbard D, Haacke EM. Quantitative flow measurements in the internal jugular veins of multiple sclerosis patients using magnetic resonance imaging. Rev Recent Clin Trials. 2012;7(2):117-126.

44. Haacke EM, Feng W, Utriainen D, et al. Patients with multiple sclerosis with structural venous abnormalities on MR imaging exhibit an abnormal flow distribution of the internal jugular veins. J Vasc Interv Radiol. 2012;23(1):60-68

45. Vachon JP, Siskin GP. Jugular interventions for multiple sclerosis and chronic cerebrospinal venous insufficiency. In: Dieter RS, Dieter R III, Dieter R Jr, Nanjundappa A, editors. Endovascular Interventions. New York, NY, USA: Springer Science+Business Media; 2014

46. Niggemann P, Seifert M, Förg A, Schild HH, Urbach H, Krings T. Positional venous MR angiography: an operator-independent tool to evaluate cerebral venous outflow hemodynamics. AJNR Am J Neuroradiol. 2012;33(2):246-251.

47. Diehn FE, Schwartz KM, Hunt CH, et al. Prevalence of incidental narrowing of the superior segment of the internal jugular vein in patients without multiple sclerosis. Clin Neuroradiol. July 2, 2013. [Epub ahead of print.]

48. Jayarman MV, Boxerman JL, Davis LM, Haas RA, Rogg JM. Incidence of extrinsic compression of the internal jugular vein in unselected patients undergoingcomputed tomography angiography. AJNR Am J Neuroradiol. 2012;33(7):1247-1250.

49. McTaggart RA, Fischbein NJ, Elkins CJ, et al. Extracranial venous drainage patterns in patients with multiple sclerosis and healthy controls. AJNR Am J Neuroradiol. 2012;33(8):1615-1620.

50. Schrauben EM, Anderson AG, Johnson K, Field A, Wieben O. Respiratory effects on phase contrast imaging of the jugular vein. J Cardiovasc Magn Res. 2012;14 Suppl 1:W4.

51. Stoquart-ElSankari S, Lehmann P, Villette A, et al. A phase-contrast MRI study of physiologic cerebral venous flow. J Cereb Blood Flow Metab. 2009;29(6):1208-1215.

52. Rodger IW, Dilar D, Dwyer J, et al. Evidence against the involvement of chronic cerebrospinal venous abnormalities in multiple sclerosis. A case-control study. PLoS One. 2013;8(8):e712495.

53. Denys BG, Uretsky BF, Reddy PS. Ultrasound-assisted cannulation of the internal jugular vein: a perspective comparison to the external landmark-guided technique. Circulation. 1993;87(5):1557-1562.

54. Lin BS, Kong CW, Tarng DC, Huang TP, Tang GJ. Anatomical variation of the internal jugular vein and its impact on temporary haemodialysis vascular access: an ultrasonographic survey in uraemic patients. Nephrol Dial Transplant. 1998;13(1):134-138. 
55. Troianos CA, Kuwik RJ, Pasqual JR, Lim AJ, Odasso DP. Internal jugular vein and carotid artery anatomic relation as determined by ultrasonography. Anesthesiology. 1996;85(1):43-48.

56. Tartière D, Seguin P, Juhel C, Laviolle B, Mallédant Y. Estimation of the diameter and cross-sectional area of the internal jugular veins in adult patients. Crit Care. 2009;13(6):R197.

57. Farina M, Novelli E, Pagani R. Cross-sectional area variations of internal jugular veins during supine head rotation in multiple sclerosis patients with chronic cerebrospinal venous insufficiency: a prospective diagnostic controlled study with duplex ultrasound investigation. $B M C$ Neurol. 2013;13(1):162.

58. Krsmanović Ž, Živković M, Lepić T, Stanković A, Raićević R, Dinćić E. Small internal jugular veins with restricted outflow are associated with severe multiple sclerosis: a sonographer-blinded, case-control ultrasound study. BMC Neurol. 2013;13(1):90.

59. Zamboni P, Menegatti E, Galeotti R, et al. The value of cerebral Doppler venous haemodynamics in the assessment of multiple sclerosis. J Neurol Sci. 2009;282(1-2):21-27.

60. Mancini M, Morra VB, Di Donato O, et al. Multiple sclerosis: cerebral circulation time. Radiology. 2012;262(3):947-955.

61. Monti L, Menci E, Ulivelli M, et al. Quantitative colour Doppler sonography evaluation of cerebral venous outflow: a comparative study between patients with multiple sclerosis and controls. PLoS One. 2011;6(9):e25012.

62. Zamboni P, Sisini F, Menegatti E, et al. An ultrasound model to calculate the brain blood outflow through collateral vessels: a pilot study. BMC Neurol. 2013;13:81.

63. Chambers B, Chambers J, Churilov L, Cameron H, Macdonell R. Internal jugular and vertebral vein volume flow in patients with clinically isolated syndrome or mild multiple sclerosis and healthy controls: results from a prospective sonographer-blinded study. Phlebology. September 24, 2013. [Epub ahead of print.]

64. Al-Omari MH, Rousan LA. Jugular vein morphology and hemodynamics in patients with multiple sclerosis. Int Angiol. 2010;29(2):115-120.

65. Bastianello S, Romani A, Viselner G, et al. Chronic cerebrospinal venous insufficiency in multiple sclerosis: clinical correlates from a multicentre study. BMC Neurol. 2011;11:132.

66. Bavera PM, Agus GB, Alpini D, et al. Results from 823 consecutive duplex exams for CCSVI in a vascular centre. Acta Phlebologica. 2012;13(3):141-148.

67. Grozdinski L, Petrov I, Iloska M. [Chronic cerebrospinal venous insufficiency diagnostics]. Gazz Med Ital Arch Sci Med. 2012;171(6): 775-783. Italian.

68. Simka M, Kostecki J, Zaniewski M, Majewski E, Hartel M. Extracranial Doppler sonographic criteria of chronic cerebrospinal venous insufficiency in the patients with multiple sclerosis. Int Angiol. 2010;29(2):109-114.

69. Lanzillo R, Mancini M, Liuzzi R, et al. Chronic cerebrospinal venous insufficiency in multiple sclerosis: a highly prevalent age-dependent phenomenon. BMC Neurol. 2013;13:20.

70. Zaniewski M, Kostecki J, Kuczmik W, et al. Neck duplex Doppler ultrasound evaluation for assessing chronic cerebrospinal venous insufficiency in multiple sclerosis patients. Phlebology. 2013;28(1): 24-31.

71. Ciciarello F, Mandolesi S, Galeandro AI, et al. Age-related vascular differences among patients suffering from multiple sclerosis. Curr Neurovasc Res. November 24, 2013. [Epub ahead of print.]

72. Baracchini C, Perini P, Calabrese M, Causin F, Rinaldi F, Gallo P. No evidence of chronic cerebrospinal venous insufficiency at multiple sclerosis onset. Ann Neurol. 2011;69(1):90-99.

73. Centonze D, Floris R, Stefanini M, et al. Proposed chronic cerebrospinal venous insufficiency criteria do not predict multiple sclerosis risk or severity. Ann Neurol. 2011;70(1):51-58.

74. Coteanu A, Gusti S, Coteanu C. The value of Doppler cerebral parameters to assess a group of patients with multiple sclerosis. Curr Health Sci J. 2013;39(1):31-34.
75. Garaci FG, Marziali S, Meschini A, et al. Brain hemodynamic changes associated with chronic cerebrospinal venous insufficiency are not specific to multiple sclerosis and do not increase its severity. Radiology. 2012;265(1):233-239.

76. Kantarci F, Albayram S, Demirci NO, et al. Chronic cerebrospinal venous insufficiency: does ultrasound really distinguish multiple sclerosis subjects from healthy controls. Eur Radiol. 2012;22(5): 970-979.

77. Radak D, Kolar J, Tanaskovic S, et al. Morphological and haemodynamic abnormalities in the jugular veins of patients with multiple sclerosis. Phlebology. 2012;27(4):168-172.

78. Weinstock-Guttmann B, Ramanathan M, Marr K, et al. Clinical correlates of chronic cerebrospinal venous insufficiency in multiple sclerosis. BMC Neurol. 2012;12:26.

79. Zivadinov R, Marr K, Cutter G, et al. Prevalence, sensitivity, and specificity of chronic cerebrospinal venous insufficiency in MS. Neurology. 2011;77(2):138-144.

80. Tromba L, Blasi S, Vestri A, Kiltzanidi D, Tartaglia F, Redler A. Prevalence of chronic cerebrospinal venous insufficiency in multiple sclerosis: a blinded sonographic evaluation. Phlebology. November 15, 2013. [Epub ahead of print.]

81. Amato MP, Saia V, Hakiki B, et al. No association between chronic cerebrospinal venous insufficiency and pediatric-onset multiple sclerosis. Mult Scler. 2012;18(12):1791-1796.

82. Auriel E, Kami A, Bornstein NM, Nissel T, Gadoth A, Hallevi H. Extra-cranial flow in patients with multiple sclerosis. J Neurol Sci. 2011;309(1-2):102-104.

83. Baracchini C, Perini P, Causin F, Calabrese M, Rinaldi F, Gallo P. Progressive multiple sclerosis is not associated with chronic cerebrospinal venous insufficiency. Neurology. 2011;77(9): 844-850.

84. Leone MA, Raymkulova O, Naldi P, et al. Chronic cerebrospinal venous insufficiency is not associated with multiple sclerosis and its severity: a blind-verified study. PLoS One. 2013;8(2):e56031.

85. Patti F, Nicoletti A, Leone C, et al. Multiple sclerosis and CCSVI: a population-based case control study. PLoS One. 2012;7(8): e41227.

86. Imperiale $\mathrm{D}$, Melis $\mathrm{F}$, Giaccone $\mathrm{C}$, et al. Chronic cerebrospinal venous insufficiency in multiple sclerosis: a sonographer-blinded case-control study. Clin Neurol Neurosurg. 2013;115(8):1394-1398.

87. Van den Berg PJ, Visser LH. The fluctuating natural course of CCSVI in MS patients and controls, a prospective follow-up. PLoS One. 2013;8(11):e78166.

88. Van den Berg PJ, Van den Berg GB, Westerhuis LW, Visser LH. Occurrence of CCSVI in patients with MS and its relationship with iron metabolism and varicose veins. Eur J Neurol. 2013;20(3):519-526.

89. Barreto AD, Brod SA, Bui TT, et al. Chronic cerebrospinal venous insufficiency: case-control neurosonography results. Ann Neurol. 2013;73(6):721-728.

90. Comi G, Battaglia MA, Bertolotto A, et al. Observational case-control study of the prevalence of chronic cerebrospinal venous insufficiency in multiple sclerosis: results from the CoSMo study. Mult Scler. 2013;19(11):1508-1517.

91. Mehrpour M, Najimi N, Fereshtehnejad SM, et al. Chronic cerebrospinal venous insufficiency in patients with multiple sclerosis: a case-control study from Iran. Perspect Med. 2012;1(1-12):375-380.

92. Tsivgoulis G, Mantatzis M, Bogiatzi C, et al. Extracranial venous hemodynamics in multiple sclerosis. A case-control study. Neurology. 2011;77(13):1241-1245.

93. Chambers B, Chambers J, Cameron H, Macdonell R. Chronic cerebrospinal venous insufficiency is not more prevalent in patients with mild multiple sclerosis: a sonographer-blinded, case-control ultrasound study. Mult Scler. 2012;19(6):749-756.

94. Doepp F, Friedemann P, Valdueza JM, Schmierer K, Schreiber SJ. No cerebrocervical venous congestion in patients with multiple sclerosis. Ann Neurol. 2010;68(2):173-183. 
95. Marder E, Gupta P, Greenberg BM, et al. No cerebral or cervical venous insufficiency in US veterans with multiple sclerosis. Arch Neurol. 2011;68(12):1521-1525.

96. Tanaka M, Uchzumi H, Tanaka K. [Evaluation of blood flow and the cross-section area of internal jugular vein in Japanese multiple sclerosis and neuromyelitis optica patients]. Rinsho Shinkeigaku. 2011;51(6):430-432. Japanese.

97. Dolic K, Weinstock-Guttman B, Marr K, et al. Risk factors for chronic cerebrospinal venous insufficiency (CCSVI) in a large cohort of volunteers. PLoS One. 2011;6(11):e28062.

98. Yamout B, Herlopian A, Issa Z, et al. Extracranial venous stenosis is an unlikely cause of multiple sclerosis. Mult Scler. 2010;6(11): 1341-1348.

99. Alpini DC, Bavera PM, Hahn A, Mattei V. Chronic cerebrospinal venous insufficiency (CCSVI) in Meniere disease: case or cause? ScienceMED. 2013;4(1):9-15.

100. Leone M, Raymkulova O, Lucenti A, et al. A reliability study of color Doppler sonography for the diagnosis of chronic cerebrospinal venous insufficiency shows low inter-rater agreement. BMJ Open. 2013;3(11):e003508.

101. Diaconu CI, Fox RJ, Grattan A, et al. Hydration status substantially affects chronic cerebrospinal venous insufficiency assessment. Neurol Clin Pract. 2013;3(5):386-391.

102. Ciccone MM, Galeandro AI, Scicchitano P, et al. Multigate quality Doppler profiles and morphological/hemodynamic alteration in multiple sclerosis patients. Curr Neurovasc Res. 2012;9(2):120-127.

103. Menegatti E, Genova V, Tessari M, et al. The reproducibility of colour Doppler in chronic cerebrospinal venous insufficiency associated with multiple sclerosis. Int Angiol. 2010;29(2):121-126.

104. Lugli M, Morelli M, Guerzoni S, Maleti O. The hypothesis of pathophysiological correlation between chronic cerebrospinal venous insufficiency and multiple sclerosis: rationale of treatment. Phlebology. 2012;27 Suppl 1:178-186.

105. Nicolaides AN, Morovic S, Menegatti E, Viseiner G, Zamboni P. Screening for chronic cerebrospinal venous insufficiency (CCSVI) using ultrasound. Recommendations for a protocol. Funct Neurol. 2011;26(4):229-248.

106. Thibault $\mathrm{P}$, Lewis W, Niblett S. Objective duplex ultrasound evaluation of the extracranial circulation in multiple sclerosis patients undergoing venoplasty of internal jugular vein stenoses: a pilot study. Phlebology. December 9, 2013. [Epub ahead of print.]

107. Simka M, Zaniewski M. Reinterpreting the magnetic resonance signs of hemodynamic impairment in the brains of multiple sclerosis patients from the perspective of a recent discovery of outflow block in the extracranial veins. J Neurosci Res. 2010;88(9):1841-1845.

108. Zamboni P, Menegatti E, Weinstock-Guttman B, et al. Hypoperfusion of brain parenchyma is associated with the severity of chronic cerebrospinal venous insufficiency in patients with multiple sclerosis: a cross-sectional preliminary report. BMC Med. 2011;9:22.
109. Zivadinov R, Poloni GU, Marr K, et al. Decreased brain venous vasculature visibility on susceptibility-weighted imaging venography in patients with multiple sclerosis is related to chronic cerebrospinal venous insufficiency. BMC Neurol. 2011;11:128.

110. Ayanzen RH, Bird CR, Keller PJ, McCully FJ, Theobald MR, Heiserman JE. Cerebral MR venography: normal anatomy and potential diagnostic pitfalls. AJNR Am J Neuroradiol. 2000;21(1): 74-78.

111. Saiki K, Tsurumoto T, Okamoto K, Wakebe T. Relation between bilateral differences in internal jugular vein caliber and flow patterns of dural venous sinuses. Anat Sci Int. 2013;88(3):141-150.

112. Gianesini S, Menegatti E, Mascoli F, Salvi F, Bastianello S, Zamboni P. The omohyoid muscle entrapment of the internal jugular vein. A still unclear pathogenetic mechanism. Phlebology. May 16, 2013. [Epub ahead of print.]

113. Radak D, Tanaskovic S, Antonic Z, Kolar J, Aleksic N, Ilijevski N. Compressive syndrome of internal jugular veins: does it matter. Phlebology. September 17, 2012. [Epub ahead of print.]

114. Coen M, Menegatti E, Salvi F, et al. Altered collagen expression in jugular veins in multiple sclerosis. Cardiovasc Pathol. 2013;22(1): 33-38.

115. Zaniewski M, Simka M. Biophysics of venous return from the brain from the perspective of the pathophysiology of chronic cerebrospinal venous insufficiency. Rev Recent Clin Trials. 2012;7(2) 88-92.

116. Shadden SC, Taylor CA. Characterization of coherent structures in the cardiovascular system. Ann Biomed Eng. 2008;36(7):1152-1162.

117. Dolic K, Siddiqui AH, Karmon Y, Marr K, Zivadinov R. The role of noninvasive and invasive diagnostic imaging techniques for detection of extra-cranial venous system anomalies and developmental variants. BMC Med. 2013;11:155.

118. Zamboni P, Menegatti E, Viselner G, Morovic S, Bastianello S. Fusion imaging technology of the intracranial veins. Phlebology. 2012;27:360-367.

119. Wattjes MP, van Oosten BW, de Graaf WL, et al. No association of abnormal cranial venous drainage with multiple sclerosis: a magnetic resonance venography and flow-quantification study. J Neurol Neurosurg Psychiatry. 2011;82(4):429-435.

120. Valk J, Van Vucht N, Pevenage P. MR venographic patterns in chronic intractable headache. Neuroradiol J. 2011;24(1):13-19.

121. Haacke EM. Abnormal MRI drainage pattern in Parkinson disease. Paper presented at the Third Annual Meeting of the International Society for Neurovascular Disease. February 23-25, 2013, Krakow, Poland.

122. Hartel M, Kluczewska E, Simka M, Ludyga T, Kostecki J, Zaniewski M. Magnetic resonance venography of chronic cerebrospinal venous insufficiency in patients with associated multiple sclerosis. Pol $J$ Radiol. 2011;76(1):59-62.
Journal of Vascular Diagnostics

\section{Publish your work in this journal}

Journal of Vascular Diagnostics is an international, peer-reviewed journal of diagnostics, focusing on non invasive vascular investigation methods involved in the evaluation of vascular diseases. The journal is committed to the rapid publication in the fields of vascular diseases. Original research, review, case reports, expert opinion and commentaries

\section{Dovepress}

are all considered for publication. The manuscript management system is completely online and includes a very quick and fair peer-review system, which is all easy to use. Visit http://www.dovepress.com/testimonials.php to read real quotes from published authors. 